\title{
XXI. Experiments on the gas from coal, chiefly with a view to its practical application
}

\section{William Henry M.D. F.R.S.}

To cite this article: William Henry M.D. F.R.S. (1819) XXI. Experiments on the gas from coal, chiefly with a view to its practical application, Philosophical Magazine Series 1, 54:256, 117-126, DOI: $10.1080 / 14786441908652197$

To link to this article: http://dx.doi.org/10.1080/14786441908652197

曲 Published online: 29 Jul 2009.

Submit your article to this journal

Џ Article views: 2

Q View related articles $\square$ 
high temperature here, and likewise the immense quantities of animal and vegetable matter continually rendered putrid by it, offensive exhalations continue to exist, that affect, particularly at night, the sense of smelling in the strongest degree, and that occasionally extend even to ships in the harbour after the land-breeze has sprung up.

[To be continued.]

XXI. Experiments on the Gas from Coal, chiefly with a view to its practical Application. By Wirliam Henry, M.D. F.R.S. $\mathbb{B O}^{\circ}$ * $^{*}$

$T$ $\mathrm{HE}$ chemical properties and composition of the gas from coal formed a principal object of two different series of experiments, the results of which I laid before the public many years ago. The first of these communications, eutitled "Experiments on the Gases obtained by the destructive Distillation of Wood, Peat, Pit-coal, Oil, Wax, \&c. with a view to the Theory of their Combustion, when employed as Sources of artificial Light," appeared in Mr. Nicholson's Philosophical Journal for June 1805 †; and the second memoir was published in the Transactions of the Royal Society for 1808 .

By the first train of experiments, I endeavoured to derive, from a careful analysis of the compound combustible gases, a measure of their illuminating power, admitting of more exact appreciation than the optical method of a comparison of shadows. The one, which I was led to propose as the most accurate, and which I still think entitled to preference, was the determination of the quantities of oxygen gas consumed, and of carbonic acid formed, by the combustion of equal measures of the different inflammable gases; that gas having the greatest illuminating power, which, in a given volume, condenses the largest quantity of oxygen. The average results of a great variety of experiments were comprised in the following table.

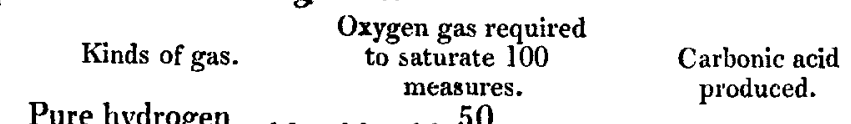

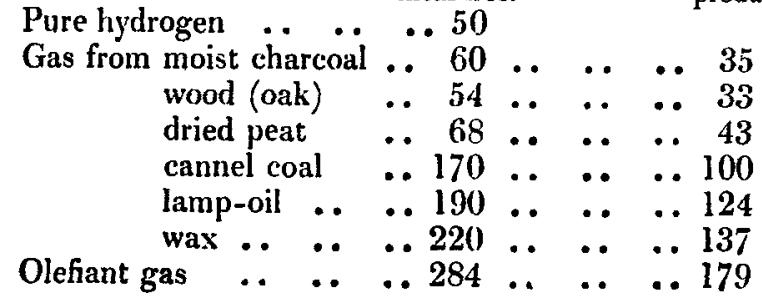

- From the Memoirs of the Literary and Philosophical Society of Manchester, vol. iii. Second Series. H 3 +8 vo Series, vol. xi. page 65 . 
In the same essay I maintained an.opinion which, on the most mature consideration, 1 see no reason to change; that the great variety of gases evolved by the destructive distillation of inflammable substanees, do not constitute so many distinct species, but are mixtures of a few, the nature and properties of which were before ascertained. It will contribute to render what follows more intelligible, if a brief account be given of those gases of known composition, the mixtures of which, in various proportions, compose, according to this view, all the observed varieties; and I shall make their comparison under a form best adapted to illustrate their praetical application.

1. Hydrogen GAS.- This is the lightest of all known gases, its specific gravity, that of atmospheric air being taken at 1000 , being about 73. As ordinarily procured, by the solution of iron or zinc in diluted sulphuric acid, it contains impurities which give it a disagreeable smell; but well purified hydrogen has little if any odour. It burns with a pale and feeble flame, not at all suited to artificial illumination.

The cubic foot weighs about 40

Product of its combustion. grains.

Consumes half a cubic foot

$$
\text { of oxygen } \ldots \frac{300}{240} \quad \text { Water .. . } 340
$$

2. Carburetted hydrogen has been shown to constitute the gas of marshes and the fire-damp of coal-mines. In these natural forms, it is contaminated with a small proportion of carbonic acid, and a larger one of azotic gas, but appears to be free from all other impurities. It is proved to be a definite compound of hydrogen and charcoal without any oxygen. It is lighter than common air, in the preportion of about 600 to 1000 ; it has very little odour; and burns with a flame greatly surpassing that of hydrogen in density and illuminating power.

Products.

oz. dr.*

1 cubic foot of carb. acid 113

A cubic foot weighs

$0 \quad 12$

Water $\quad \ldots \quad \ldots \frac{19}{36}$

feet of oxygen

$\frac{210}{36}$

3. Carbonic oxide is rather lighter than common air. It contains no hydrogen, and is purely a compound of charcoal and oxygen, the latter being in just half the proportion which is required to constitute carbonic acid. It burns with a feeble blue light.

* The avoirdupois ounce of $437 \frac{1}{2}$ grains, or 16 drachms, is to be understood. 
Product.

oz. dr. oz. dr.

A cubic foot weighs $\quad . . \quad 1 \quad 3$

Consumes $\frac{r}{2}$ a cubic foot

of oxygen $\quad \ldots \quad \ldots 0011$

l 14 Carbonic acid 114

4. Olefiant gas, or Bi-carburetted hydrogen.-This has been demonstrated to be a compound of nearly 85 by weight charcoal, and 15 hydrogen, without any oxygen. It is a little lighter than common air, viz. in the proportion of about 974 to 1000. It surpasses all other gases in the brightness and density of its flame. Its name was originally derived from the property which it possesses, of being speedily and entirely condensed, by rather more than an equal volume of chlorine gis, into a liquid resembling oil in appearance, but since shown to approach more nearly to the nature of ether.

Products.

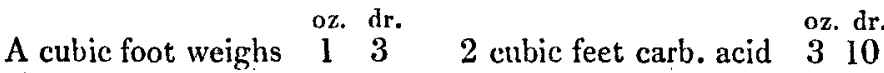

Consumes 3 cubic

$\begin{array}{lllllllll}\text { feet of oxygen } & . & \frac{4}{5} & 0 \\ 5 & 3 & & \text { Water } & \ldots & \ldots & \ldots & \frac{1}{9}\end{array}$

Olefiant gas I found to be one of the products of the distillation of oil and of bees' wax, and was led, therefore, to suggest, that the wick of a lamp or candle, surrounded by flame, is to be considered as a bundle of ignited capillary tubes, into which the melted inflammable matter is drawn, and there resolved, not into a condensable vapour, but into olefiant and carburetted hydrogen gases. In the gas from coal, also, I detected the presence of olefiant gas, by the test of the action of chlorine.

In the second series of experiments *, I submitted to distillation, on a small scale, various kinds of coal, from different parts of the kingdom. The aëriform products, at different stages of the process, were kept apart, and were separately analysed. From coal distilled in small iron tubes or retorts, which, when filled, were placed at once in a low red heat, small quantities of sulphuretted hydrogen and carbonic acid gases came over at first, in mixture with the other gases, but in a gradually diminishing proportion, till at length, in the last products, they were not discoverable at all. The production of olefiant gas observed the same order, and a gradual diminution took place, as the process advanced, in the combustibility of the gas, as determined by its requiring less and less oxygen for saturation. A great variety was

* Phil. Trans. 1808, page 282. 
ascertained to exist in the quality of the gas from different kinds of coal; that from Wigan cannel holding the highest rank in illuminating power, and that from the stone coal of South Wales the lowest.

Since the period when the second of these papers was published, the use of artificial gases, as a source of light, has been rapidly increasing in this, and, I believe, in other countries, and promises to attain an extent and importance sufficient to justify uny labour that may tend, however remotely, to its improved application. It has frequently happened, of late years, that I have been requested by the proprietors of large manufactories lighted by gas in this neighbourliood, to give an opinion on practical points, respecting some of which I felt myself incompetent to decide, from the want of the necessary data. It is to supply these data, that I have once more returned to the investigation of the subject. The objects which I have had it in view to determine by the following course of experiments, are, whether, on the large scale of manufacture, there is a decline in the value of the aëriform products of coal, from the beginning to the end of the distillation, similar to that which takes place on a small scale; -at what stages of the process those gases; which may be considered as impurities, are chiefly evolved; and whether they are essential or accidental products;-whether the method of removing the sulphuretted hydrogen and carbonic acid gases by quicklime, which I suggested in the second memoir, is adequate to the complete purification of coal gas;-whether this purification is attended with any loss of that portion of the gas which, on account of its superior illuminating power, it is desirable not to remove;-and, if such a loss should be found to ensue, whether it may not be avoided by some modification of the purifying process. In determining these points, I was indebted for the necessary supplies of gas to $\mathrm{Mr}$. Lee, at whose extensive manufactory the principal facts were ascertained, that formed the basis of the first accurate calculations respecting the oconomy of gas from coal.*

\section{On the Quality of the Gas, at different Stages of the Distilla- tion.}

The gas which I first submitted to experiment was obtained from Wigan cannel coal, a substance preferred in this neighbourhood as affording aëriform products, which, both by their quantity and quality, more than compensate its higher price $\dagger$. The retorts are charged while red hot with this substance, and indeed

* See Mr. Murdoch's " Account of the Application of the Gas from Coal to ceconomical Purposes," Phil. Trans. 1808, page 124.

$\uparrow$ About a shilling per cwt. of $112 \mathrm{lb}$. or $13 \frac{1}{2} d$. delivered in Manchester. 


\section{Experiments on the Gas from Coal.}

are never suffered, during the whole of the winter season, to fall below the temperature of ignition. The gas was collected in a bladder furnished with a stop-cock, which was fixed into an opening in the pipe between the retort and the tar-pit. It was taken at this place, in order to avoid contact with water, and admixture with any atmospherical air, that might accidentally remain in the gasometer. Wishing to examine the gas in a perfectly recent state, and finding it impossible to make the necessary experiments with sufficient accuracy in a shorter interval, I was obligt $d$ to be satisfied with procuring it every other hour. In this place, I shall only state the general results ; and I shall describe, in a subsequent part of the paper, the methods of analysis, in order that other persons, who may choose to compare my experiments with their own, may conduct them under equal circumstances.

By the expression impure gas, is to be understood, the gas precisely in the state in which it was collected from the retort; and by purified gas, the same product after being freed from carbonic acid and sulphuretted hydrogen by solution of pure potash, applied in very small quantity, relatively to the volume of the gas, and with the least agitation adequate to the effect.

TABLE I.

Showing the Quality of Gas from 1120 lls. of Cannel, at different Periods of the Distillation.

Hours from the 100 measures of im- $100 \mathrm{~m}$. puritied gas $100 \mathrm{~m}$. of purified commence- pure gas contain of ment. $\overbrace{\text { Sul. hyd. carb. ac. }} \overbrace{\text { Olef. other infl. az. }}$ \begin{tabular}{l|l}
\multicolumn{2}{|c}{ gas } \\
cons. & give \\
oxyg. & carb. \\
& acid.
\end{tabular}

\begin{tabular}{cc|}
$\frac{1}{2}$ & an hour \\
1 & hour .. \\
3 & hours .. \\
5 & hours .. \\
7 & hours .. \\
9 & hours .. \\
$10 \frac{1}{2}$ & hours .. \\
12 & hours ..
\end{tabular}

\begin{tabular}{l|l}
$0 \frac{1}{2}$ & $5 \frac{I}{2}$ \\
3 & $3 \frac{I}{2}$ \\
$2 \frac{1}{2}$ & $2 \frac{I}{2}$ \\
$2 \frac{I}{2}$ & $2 \frac{I}{2}$ \\
2 & $2 \frac{I}{2}$ \\
$0 \frac{I}{2}$ & $2 \frac{I}{2}$ \\
0 & 2 \\
0 & $0 \frac{I}{2}$
\end{tabular}

\begin{tabular}{|c|c|}
16 & 64 \\
15 & $77 \frac{1}{4}$ \\
15 & 80 \\
13 & 72 \\
9 & 76 \\
8 & 77 \\
6 & 74 \\
4 & 76
\end{tabular}

20

$180 \quad 94$

$210: 112$

$200 \quad 108$

$15 \quad$\begin{tabular}{l|r|r}
176 & 94
\end{tabular}

$15 \quad 170 \quad 83$

$15 \quad 150 \quad 73$

$20 \quad 120 \quad 54$

Excluding from the calculation the azotic gas, with various proportions of which the products were contaminated, the following table shows the quantity of oxygen gas consumer, and of carbonic acid produced, by the really combustible part of the gas. 
TABI E II.

Showing the Quality of the really combustible Part of the Gas, at different Periods of Distillation.

100 measures of

Take oxygen.

Give carb. acid.

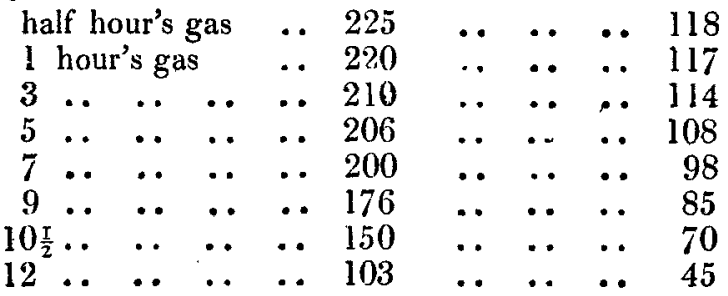

The next set of experiments was made on gas from common coal, got atClifton near Manchester, and of fair average quality.

TABLE III.

Showing the Quality of the Gas from 1120 lbs. of common Coal, at different Periods of the Distillation. $100 \mathrm{~m}$. of im- $\eta^{\prime} 100$ measures of $\mid 100$ measures pure gas con- purified gas. purified.

1 hour's gas $\mid$\begin{tabular}{c|c|c|c|c|c|c|c|c|c|} 
& hyd. & 3 & & acid. & 10 & 90 & 0 & 164 & 91
\end{tabular}

\begin{tabular}{ll|l|l|l|l|l|l|l}
3 hours do. & 2 & 2 & 9 & 91 & 0 & 168 & 93
\end{tabular}

\begin{tabular}{l|l|l|l|l|l|l|l}
5 hours do. & 3. & 2 & 6 & 94 & 0 & 132 & 70
\end{tabular}

\begin{tabular}{|l|l|l|l|l|l|l|l}
7 hours do. & 1 & 3 & 5 & 80 & 15 & 120 & 64
\end{tabular}

\begin{tabular}{rl|l|l|l|l|r|r|r}
9 & hours do. & 1 & $2 \frac{1}{2}$ & 2 & 89 & 9 & 112 & 60 \\
11 & hours do. & 1 & 1 & 0 & 85 & 15 & 90 & 43
\end{tabular}

Exclusive of the azote, with which the three last portions of gas were mingled, they consumed oxygen and gave carbonic acid as follows. The seven hours gas in this instance, as sometimes happens from irregularities of temperature, was more combustible than that collected two hours sooner.

Consumed oxygen. Gave carb. acid.

$$
\begin{aligned}
& 100 \mathrm{~m} \text {. of } 7 \text { hours gas } \quad \ldots \quad 140 \quad \ldots \quad \ldots 75
\end{aligned}
$$

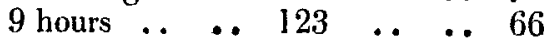

$$
\begin{aligned}
& 11 \text { hours } \ldots . . \quad 106 \quad \ldots \quad \ldots 50
\end{aligned}
$$

A comparison of the results exhibited in the third table, with those of the distillation of cannel coal, is greatly in favour of the latter substance as a source of light. This will appear most distinctly, by setting against each other the proportions of oxygen, which are consumed by the gases evolved from the two substances at equal times from the commencement. 
TABIE IV.

Comparative Table of the Qualilies of the Gases from Wigan Cannel, and from common Coal, at equal Times from the Commencement of the Distillation.

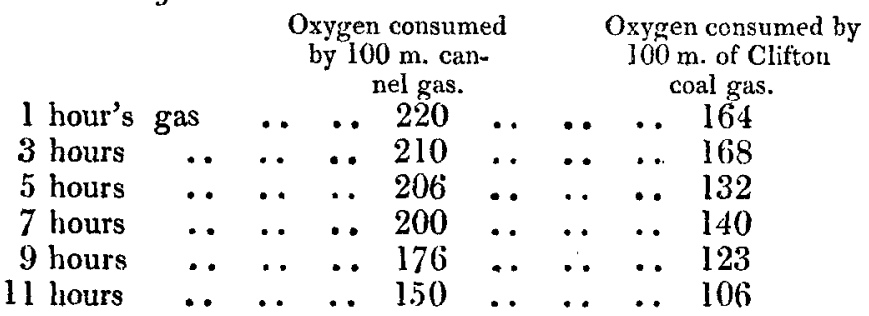

It appears from these experiments, that the gas from cannel has, in an equal volume, an illuminating power about one-third greater than that from coal of medium quality. The quantity, alșo, from the former substance, exceeded by about one-seventh that obtained from coal, distilled under precisely similar circumstances; 3500 cubic feet of gas having been collected from 1120 pounds of cannel, and only 3000 cubic feet from the same quantity of coal. The whole product of one distillation of cannel, mixed together in a gasometer, was of such quality, that 100 measures required for combustion 155 measures of oxygen gas, and gave 88 measures of carbonic acid. But as the gas was contaminated with 15 measures of azote in every hundred, the oxygen, required for saturating 100 measures of the really combustible part of it, may be stated at 195 ; and the carbonic acid produced at 110 . It may be necessary to observe, that in comparing the value of gases produced from different kinds of coal, or from the same kind of coal differently treated, it is rot enough to determine the quantity of aerriform products; and no satisfactory conclusion can be drawn respecting the relative fitness of any variety of coal for affording gas, or the advantages of different modes of distillation, unless the degrees of combustibility of the gases compared be determined, by finding experimentally the proportion of oxygen gas required for their saturation.

The results expressed in the first table, when contrasted with those which I formerly obtained by the destructive distillation of small quantities of coal, present several circumstances of disagreement, as to the quality of the products at different stages of the operation. In small experiments, the sulphuretted hydrogen and carbonic acid gases were evolved only at the early stages of the process; and sulphuretted hydrogen, especially, could not by the nicest tests be discovered in the last products of gas. On the large scale, both these gases continue to be evolved throughout the whole operation, though in greatly diminished proportion to- 
wards the latter end. Even in the advanced stages of large distillations, the presence of sulphuretted hydrogen in coal-gas may be traced by the proper test, though not in a quantity that admits of being easily measured. The test which I used for some time was the white oxide of bismuth, for which $I$ afterwards substituted white lead, ground with a little water to the proper consistence, and spread by a camel's hair pencil on a slip of card. 'This was secured by a small pair of forceps fixed in a cork, by means of which the slip of card could be placed in a jar or bottle of the gas, and kept there for some time. By experiments on artificial mixtures, I found that a cubic inch of sulphuretted hydrogen, diffused through twenty thousand cubic inches of common air, distinctly affected the test, which it changed to a light yellowish or straw colour. By mixing sulpliuretted hydrogen with various proportions of common air, I prepared coloured cards of a variety of shades, which served as standards of comparison for judging of proportions of sulphuretted hydrogen in coal-gas, which were too ninute to be accurately ineasured.

In the small experiments made several years ago, I'never found, in the early products of gas from cannel coal, a proportion of olefiant gas at all approaching that which is noted in Table I. and its quantity in small distillations rapidly decreased, until in the latter products it could be no longer traced at all. The method of analysis, which I formerly employed, led me, however, as I have lately discovered, to under-rate the proportion of olefiant gas, aud to over-estimate that of sulphuretted hydrogen. But making due allowance for this error, the superiority of the products of large operations, so far as respects olefiant gas, still exists, and is confirmed by comparative experiments on a small scale which I have lately made. Thus it appears from Table I. that even after twelve hours continuance of the process, olefiant gas still constitutes 4 per cent. of the gases evolved from cannel. The other inflammable gases, also, when obtained in large quantity, are more uniform in quality, and possess, towards the close of the process, much greater combustibility and illuminating power, than when procured in smail experiments. This superiority is obviously dependent on the greater facility of preserving an uniform temperature, in all chemical processes which are carried on upon a scale of magnitude.

The temperature to which the coal is subjected, must necessarily be a point of the greatest importance to the quantity and quality of the aëriform products ; for while too low a heat distils over, in the form of a condensible fluid, the bituminous part of the coal which ought to afford gas, too high a temperature, on the contrary, occasions the production of a large relative proportion of the lighter and less combustible gases. It would be a 
great step in the improvement of the manufacture of coal-gas, if the whole of the hydrogen could be obtained in combination with that proportion of charcoal which constitutes olefiant gas ; and it is satisfactory to know, that no impediment to this arises out of the proportion of the hydrogen and charcoal present in coal. If this object be ever accomplished, it will probably be by the discovery of means of uniformly supporting such a temperature as shall be adequate to the production of olefiant gas, and shall never rise above it ; and some probability of success is perhaps derivable from the fact, that $M$. Berthollet, by the careful decomposition of oil, which in my experiments afforded a mixture of gases, succeeded in obtaining olefiant gas in a state of purity*.

With the view of ascertaining how low a degree of heat is adequate to the production of gas from coal, I placed a small iron retort, containing cannel, in melted solders of various composition, without obtaining more than the common air of the vessel. The retort charged with fresh materials, was then immersed in melted lead; but after expelling the common air, no more than a few bubbles of gas came over, and that only when the lead, by being kept over the fire, had acquired a temperature about its fusing point. On restoring this temperature by adding fresh metal, the evolution of gas was always suspended. I placed also one of $\mathrm{Mr}$. Wedgwood's pyrometer pieces in contact with a retort which was at work at Mr. Lee's manufactory, and which showed only a dull red or blood-coloured heat; but, after remaining in that situation half an hour, a contraction of barely one degree of the scale had taken place. This temperature, however, I suspect is rather too low, and has a tendency to distil over too much tar, and consequently to produce less gas than might be obtained by a degree of heat somewhat higher. The best adapted temperature will probably be found to vary with different kinds of coal ; and I have been prevented from ascertaining it with respect to cannel, by the inconveniences that would arise from disturbing the regulai arrangements of a large manufactory. From some experiments of Mr. Brande, it appears that the sudden application of the requisite heat evolves from coal much more gas, than the gradual heating of a cool retort up to the point of ignition $\uparrow$.

In the experiments upon gas from Wigan cannel, the results of which are comprised in the first table, azotic gas was found in all the aëriform products, from the beginning to the end of the

* Mémoires de la Soc. d'Arcueil, ii. 84.

$\uparrow$ Journal of Science, vol. i. page 75.

operation. 
operation. But in experiments on the gas obtained at other times from the same substance, no appreciable quantity of azotic gas could be discovered till after the sixth hour of the process, when it began to appear, and progressively rose to 20 parts in the hundred. Of this purity of the early products from azote, and appearance of it in the latter ones, Mr. Dalton was an eye witness on one occasion, when he was so good as to co-operate with me; and I had afterwards repeated opportunities of verifying the fact. With the view of ascertaining whether the azote found its way from the atmosphere into the distilling vessels, I subjected 100 grains of cannel coal to heat in a glass retort, the capacity of whose body and neck did not together exceed $1 \frac{1}{4}$ cubic inch. Besides a portion of gas which was lost, 50 cubic inches were collected, which, on careful analysis, were found to contain 5 cubic inches of azotic gas. Of these only one cubic inch can be traced to the common air present in the retort at the outset; and the other 4 cubic inches must have been furnished by the coal itself.

It is resonable indeed to expect, that a substance like coal, which affords ammonia under some circumstauces, should, under others, yield the elements of that alkali in a detached state ; and the reason, why azote is for the most part not to be found in the gas which is first evolved, is, that at a low temperature, that element unites with hydrogen, and composes ammonia. But when the contents of the retort, which, for some time, have been kept comparatively cool by the escape of condensable fluids, become more intensely heated, ammonia is either not formed, or, if formed, is decomposed again into azotic and hydrogen gases, both of which may be traced in the aëriform products of the advanced stages of distillation. As a matter of practice, it is certainly desirable that the azote existing in coal should enter into the composition of a condensable fluid, rather than that it should escape in a gaseous state; for it is an impurity which, when onee mingled with the combustible gas, cannot be removed by any known method, and must materially impair its illuminating power. That such an effect must result from its presence, may be inferred from the experiments of Sir H. Davy, who found that an explosive mixtlire of carburetted hydrogen and common air was deprived of its combustibility by being mixed with one sixth of its bulk of azotic gas*.

[To be continued.]

* On the Safety-lamp, page 30 . 